\title{
Procedure Guideline for Myocardial Perfusion Imaging $3.3^{*}$
}

\author{
H. William Strauss ${ }^{1}$, D. Douglas Miller ${ }^{2}$, Mark D. Wittry ${ }^{2}$, Manuel D. Cerqueira ${ }^{3}$, Ernest V. Garcia ${ }^{4}$, \\ Abdulmassi S. Iskandrian ${ }^{5}$, Heinrich R. Schelbert ${ }^{6}$, Frans J. Wackers ${ }^{7}$, Helena R. Balon ${ }^{8}$, Otto Lang ${ }^{9}$, \\ and Josef Machac ${ }^{10}$
}

\begin{abstract}
${ }^{I}$ Memorial Sloan Kettering Cancer Center, New York, New York; ${ }^{2}$ Saint Louis University Health Sciences Center, St. Louis, Missouri; ${ }^{3}$ Georgetown University Medical Center, Washington, DC; ${ }^{4}$ Emory University Hospital, Atlanta, Georgia; ${ }^{5}$ Allegheny University of the Health Sciences, Philadelphia, Pennsylvania; ${ }^{6}$ UCLA School of Medicine, Los Angeles, California; ${ }^{7}$ Yale University School of Medicine, New Haven, Connecticut; ${ }^{8}$ William Beaumont Hospital, Royal Oak, Michigan; ${ }^{9}$ Third Medical School, Charles University, Prague, Czech Republic; and ${ }^{10}$ Mount Sinai School of Medicine, New York University, New York
\end{abstract}

\section{PURPOSE}

The purpose of this guideline is to assist nuclear medicine practitioners in recommending, performing, interpreting, and reporting the results of myocardial perfusion imaging studies.

\section{BACKGROUND INFORMATION AND DEFINITIONS}

Myocardial perfusion imaging uses an intravenously administered radiopharmaceutical to depict the distribution of blood flow in the myocardium. Perfusion imaging identifies areas of relatively reduced myocardial blood flow associated with ischemia or scar. The relative regional distribution of perfusion can be assessed at rest, during cardiovascular stress, or both. Imaging can also be performed during acute events (e.g., with tracer injection during chest pain such as in the critical care unit or emergency department). Perfusion images can be recorded with planar or tomographic single-photon or positron imaging techniques, using radiopharmaceuticals that are extracted and retained for a variable period of time by the myocardium. The data can be analyzed by visual inspection or by quantitative techniques. Some Food and Drug Administration (FDA)-approved radiopharmaceuticals used for myocardial perfusion imaging include ${ }^{201} \mathrm{Tl}$ and the ${ }^{99 \mathrm{~m}} \mathrm{Tc}$-labeled radiopharmaceuticals such as sestamibi or tetrofosmin for single-photon imaging as well as ${ }^{13} \mathrm{~N}$-ammonia and ${ }^{82} \mathrm{Rb}$ for PET imaging.

Patients with significant coronary artery narrowing or abnormal coronary flow reserve will have a zone of diminished radiopharmaceutical concentration in the area of relatively decreased perfusion. If either the area or the severity

\footnotetext{
Received Jul. 29, 2008; revision accepted Jul. 30, 2008.

For correspondence or reprints contact: H. William Strauss, Memorial Sloan-Kettering Cancer Center, 1275 York Ave., Room S-212, New York, NY 10021.

E-mail: straussh@mskcc.org

*YOU CAN ACCESS THIS ACTIVITY THROUGH THE SNM WEB SITE (http://www.snm.org/guidelines).

COPYRIGHT @ 2008 by the Society of Nuclear Medicine, Inc.

DOI: $10.2967 /$ jnmt.108.056465
}

of decreased tracer concentration is worse when the tracer is administered during stress than at rest, the zone of decreased tracer concentration is most likely due to ischemia. If the area of diminished tracer concentration remains unchanged from rest to stress, the defect most likely represents scar, although in some cases, it may represent viable, underperfused myocardium. (Such fixed abnormalities may also represent high-grade obstruction to zones of viable, hibernating myocardium. When ${ }^{201} \mathrm{Tl}$ is used as the radiopharmaceutical, redistribution of tracer on delayed images may be useful to distinguish these lesions from scar. When the ${ }^{99 \mathrm{~m}} \mathrm{Tc}$-labeled radiopharmaceuticals are used, administering nitroglycerin before injection at rest may help make this distinction by improving perfusion (and tracer uptake) in the severely ischemic but viable region. Patients who fail to demonstrate myocardial viability with conventional SPECT techniques may benefit from ${ }^{18} \mathrm{~F}$ FDG PET, particularly those patients with marked left ventricular dysfunction.) In addition to regional perfusion, recording myocardial perfusion data with electrocardiographic (ECG) gating permits calculation of global and regional ventricular function and assessment of the relationship of perfusion to regional function.

\section{PROCEDURES}

\section{A. Patient Preparation}

1. Patients should be fasting before rest myocardial perfusion imaging for at least $4 \mathrm{~h}$. Cardiac medications should be withheld if the examination is performed to detect coronary disease. Cardiac medication should be taken as usual when the examination is performed to determine the effectiveness of medical therapy. Radiopaque objects in the area of the thorax should be removed before imaging; implanted radiopaque objects (metal, silicone, etc.) should be noted as potential attenuators of cardiac activity. In patients with severe coronary disease, it may be advisable to administer 
nitroglycerin sublingually about $3 \mathrm{~min}$ before rest injection of the radiopharmaceutical.

2. Graded exercise stress is usually performed with a treadmill or bicycle ergometer with continuous patient monitoring. All stress procedures must be supervised by a qualified health care professional and performed in accordance with the guidelines of the American Heart Association and American College of Cardiology. A fasting state is recommended for a minimum of $4 \mathrm{~h}$ before the stress study. In general, patients undergoing a stress study should be hemodynamically and clinically stable for a minimum of $48 \mathrm{~h}$ before testing. Although patients who are unable to exercise for noncardiac reasons (e.g., severe pulmonary disease, arthritis, amputation, or neurologic disease) may be stressed pharmacologically with drugs designed to cause coronary hyperemia or increased cardiac work (Table 1), exercise stress is preferred because it provides additional prognostic information.

If not medically contraindicated, it is recommended that medications such as calcium channel blocking drugs and $\beta$-blocking drugs that may alter the heart rate and blood pressure response to exercise be withheld on the day of diagnostic studies. A secure intravenous line should be established for the administration of the radiopharmaceutical during stress. Patients undergoing exercise stress should wear suitable clothing and shoes.

3. Two types of pharmacologic stress are useful to evaluate myocardial perfusion.

a. Vasodilator stress agents may be administered to create coronary hyperemia (i.e., dipyridamole or adenosine). Caffeine-containing beverages and methylxanthine-containing medications that interfere with the coronary hyperemia produced by these drugs should be discontinued for at least $12 \mathrm{~h}$ before pharmacologic stress imaging (longer for long-acting methylxanthine preparations). When possible, patients should also undergo low-level exercise to minimize symptoms associated with the

TABLE 1

Modalities for Stress Testing

\begin{tabular}{ll}
\hline Type of stress & \multicolumn{1}{c}{ Modality } \\
\hline Exercise & Submaximal \\
& Symptom limited \\
& Maximal \\
\hline Pharmacologic & Vasodilators \\
& Adenosine \\
& Dipyridamole \\
& Inotropic \\
& Dobutamine \\
& Dobutamine plus atropine \\
\hline & \\
\hline${ }^{*}$ Review history for atropine contraindications. \\
\hline
\end{tabular}

vasodilators, maintain blood pressure, and minimize subdiaphragmatic tracer uptake. When dipyridamole is used as the vasodilator, aminophylline (or a caffeinated beverage) may be administered after administration of the radiopharmaceutical to reverse the effects of the vasodilator. This is usually not required for adenosine, because of its short duration of action, but rarely aminophylline is necessary when symptoms persist even after cessation of adenosine.

b. Ino/chronotropic agents may be administered to increase myocardial oxygen demand. Drugs that may blunt the chronotropic response to adrenergic stimulant stress with dobutamine (i.e., $\beta$-adrenergic blocking agents) should be discontinued before the procedure if possible. In some patients, atropine may be required to increase the heart-rate response, although increasing the heart rate by atropine does not cause the same increment in coronary blood flow as does a similar increment in heart rate induced by exercise. Patients should be fasting for a minimum of $4 \mathrm{~h}$ before pharmacologic stress testing. Although dobutamine is in routine clinical use for pharmacologic stress, it is not an FDAapproved indication for the drug.

\section{B. Information Pertinent to Performing the Procedure}

A cardiovascular medical history and cardiorespiratory examination, including baseline vital signs, should be obtained before stress studies. Specific areas in the medical history requiring attention are the indication for the examination, medications, symptoms, cardiac risk factors, and prior diagnostic or therapeutic procedures. Special attention must be directed to determine whether the patient may have an unstable coronary syndrome, obstructive hypertrophic cardiomyopathy, or aortic valve stenosis that could increase the risk of stress. Patients should also be assessed for obstructive airway disease that could increase the risk of bronchospasm with infusion of dipyridamole or adenosine. A 12-lead electrocardiogram should be reviewed for evidence of acute ischemia, arrhythmia, or conduction disturbances, that is, left bundle branch block, before stress myocardial perfusion imaging. Diabetic patients requiring insulin should be evaluated on a case-by-case basis to optimize diet and insulin dosing on the day of the examination. In patients with left bundle branch block, it is useful to use vasodilator stress to minimize the effect of the abnormal septal wall motion on the myocardial image of "perfusion."

\section{Precautions/Contraindications}

1. Contraindications to exercise testing are unstable angina with recent $(<48 \mathrm{~h})$ angina or congestive heart failure, documented acute myocardial infarction (MI) within 2-4 d of testing, uncontrolled systemic (systolic $>220 \mathrm{~mm} \mathrm{Hg}$, diastolic $>120 \mathrm{~mm} \mathrm{Hg}$ ) or pulmonary hypertension, untreated life-threatening arrhythmias, 
uncompensated congestive heart failure, advanced atrioventricular block (without a pacemaker), acute myocarditis, acute pericarditis, severe mitral or aortic stenosis, severe obstructive cardiomyopathy, and acute systemic illness. Relative contraindications to exercise stress include conditions that may interfere with exercise, such as neurologic disease, orthopedic disease, arthritic disease, severe pulmonary disease, peripheral vascular disease, severe deconditioning, or inability to comprehend the exercise protocol.

2. Patients with a history of severe bronchospasm, pulmonary disease (i.e., asthma or pulmonary hypertension), prior intubation for severe pulmonary disease, systemic hypotension (BP systolic $<90 \mathrm{~mm} \mathrm{Hg}$ ), severe mitral valve disease, and prior hypersensitivity to dipyridamole or adenosine should not undergo vasodilator stress with dipyridamole or adenosine. Patients requiring methylxanthine-containing medications to control their bronchospasm should not be tested with vasodilator agents. Ino/chronotropic agents may be used in these patients. Patients with mild bronchospasm may undergo vasodilator stress testing, particularly after pretreatment with a bronchodilator such as an albuterol inhaler.

Patients with advanced (second- or third-degree) atrioventricular block or sick sinus syndrome should not be tested with adenosine because of its negative dromotropic (SA + AV node) effect. Additional contraindications to vasodilator agents include severe aortic stenosis, severe obstructive hypertrophic cardiomyopathy, and severe orthostatic hypotension. The use of dipyridamole or adenosine is not recommended in pregnant or lactating women.

3. Ino/chronotropic agents are contraindicated in patients with ventricular tachyarrhythmias. These agents should be used with caution in patients with unstable angina, obstructive or hypertrophic cardiomyopathy, and early after acute infarction.

4. Life support instrumentation and emergency drugs must be available in the immediate vicinity of the stress laboratory. A physician or other trained medical personnel currently certified in advanced cardiac life support must be immediately available during the stress and recovery phases. Continuous ECG monitoring must be performed during the stress and recovery phases. Vital signs (heart rate and blood pressure) and a 12-lead electrocardiogram should be recorded at regular intervals throughout the stress and recovery phases. The patient should be questioned at regular intervals (e.g., every 1-2 min) for symptoms of myocardial ischemia or the side effects of pharmacologic stress agents using a standardized scale (e.g., $1=$ very light to $10=$ most severe). Patients with implanted defibrillator devices may require temporary adjustment of their device to prevent stress-induced triggering. Failure of monitoring equipment is an absolute indication to stop exercise.
5. It is mandatory that all Occupational Safety and Health Administration, Nuclear Regulatory Commission, and state regulatory guidelines for the safe handling of syringes, needles, radioactive materials, and patient waste be followed at all times.

\section{Radiopharmaceuticals}

The following single-photon-emitting radiopharmaceuticals are FDA-approved for use as myocardial perfusion tracers: ${ }^{201}$ Tl-chloride, ${ }^{99 \mathrm{~m}} \mathrm{Tc}$-sestamibi, and ${ }^{99 \mathrm{~m}} \mathrm{Tc}$-tetrofosmin. The following positron-emitting radiopharmaceuticals are approved for use as myocardial perfusion tracers: ${ }^{82} \mathrm{Rb}$ and ${ }^{13} \mathrm{~N}$-ammonia. FDA recommendations for the maximum administered dose for a combined rest and stress study (performed on the same day) are a total of $148 \mathrm{MBq}(4 \mathrm{mCi})$ of ${ }^{201} \mathrm{Tl}, 1,480 \mathrm{MBq}$ (40 $\mathrm{mCi}$ ) of ${ }^{99 \mathrm{~m}} \mathrm{Tc}$-labeled radiopharmaceuticals, $4,440 \mathrm{MBq}(120 \mathrm{mCi})$ of ${ }^{82} \mathrm{Rb}$, and up to 1,480 $\mathrm{MBq}(40 \mathrm{mCi})$ of ${ }^{13} \mathrm{~N}$ ammonia. When rest and stress studies are performed on separate days, the dose of thallium may be $140 \mathrm{MBq}(4 \mathrm{mCi})$ for each injection and the dose of technetium-labeled agents may be $1,110 \mathrm{MBq}(30 \mathrm{mCi})$ for each injection. Doses may be adjusted at the discretion of the prescribing physician (Table 2).

\section{E. Image Acquisition: Single Photon}

Data can be acquired using planar imaging, SPECT, or a combination of both techniques. SPECT should be performed whenever possible.

1. Planar images are usually recorded with the patient supine on the imaging table in at least 3 standard views: anterior view, a left anterior oblique view to optimize visualization of the septum (usually $45^{\circ}$ ), and a left lateral view (preferably recorded with the patient in the right lateral decubitus position to minimize attenuation from the abdomen). After injection of thallium at peak exercise stress, image acquisition should commence as soon as the patient's heart rate has recovered to near baseline values (preferably within 10 min of injection). After injection of a ${ }^{99} \mathrm{~m}$ Tc-labeled tracer with vasodilators, stress imaging may start in 30-60 min. Additional views may be required to account for unusual cardiac orientation within the thorax. Acquisition is performed with a $\gamma$-camera equipped with either a lowenergy all-purpose or high-resolution parallel-hole collimator, with the camera as close to the chest as possible. Images should be acquired in such a fashion that the heart occupies approximately $35 \%-50 \%$ of the usable field of view (using magnification during image acquisition if a large-field-of-view camera is used to record the data). Images of diagnostic quality can be obtained if a minimum of 500,000 counts is recorded in each view (a minimum of 400 counts $/ \mathrm{cm}^{2}$ of normal myocardium). The timing of imaging after injection of the radiopharmaceutical will vary with the radiophar- 
TABLE 2

Radiation Dosimetry for Adults*

\begin{tabular}{|c|c|c|c|}
\hline Radiopharmaceutical & $\begin{array}{c}\text { Intravenously } \\
\text { administered activity }\end{array}$ & $\begin{array}{l}\text { Organ receiving the largest } \\
\text { radiation dose }\end{array}$ & Effective dose \\
\hline${ }^{201} \mathrm{Tl}^{-c h l o r i d e}{ }^{\dagger}$ & $74-148 \mathrm{MBq}(2-4 \mathrm{mCi})$ & $\begin{array}{l}\text { Kidneys, } 0.46 \mathrm{mGy} / \mathrm{MBq} \\
\quad(1.7 \mathrm{rad} / \mathrm{mCi})\end{array}$ & $0.23 \mathrm{mSv} / \mathrm{MBq}(0.85 \mathrm{rem} / \mathrm{mCi})$ \\
\hline 99mTc-sestamibi ${ }^{\ddagger}$ & $740-1,480 \mathrm{MBq}(20-40 \mathrm{mCi})$ & $\begin{array}{l}\text { Gallbladder, } 0.039 \mathrm{mGy} / \mathrm{MBq} \\
\quad(0.14 \mathrm{rad} / \mathrm{mCi})\end{array}$ & $0.0085 \mathrm{mSv} / \mathrm{MBq}(0.031 \mathrm{rem} / \mathrm{mCi})$ \\
\hline 99mTc-tetrofosmin & $740-1,480 \mathrm{MBq}(20-40 \mathrm{mCi})$ & $\begin{array}{l}\text { Gallbladder, } 0.031 \mathrm{mGy} / \mathrm{MBq} \\
\text { (0.11 rad } / \mathrm{mCi})\end{array}$ & $0.0067 \mathrm{mSv} / \mathrm{MBq}(0.025 \mathrm{rem} / \mathrm{mCi})$ \\
\hline${ }^{82} \mathrm{Rb} \S$ & $1,100-1,850 \mathrm{MBq}(30-50 \mathrm{mCi})$ & $\begin{array}{l}\text { Kidneys, } 0.018 \mathrm{mGy} / \mathrm{MBq} \\
(0.067 \mathrm{rad} / \mathrm{mCi})\end{array}$ & $0.0048 \mathrm{mSv} / \mathrm{MBq}(0.018 \mathrm{rem} / \mathrm{mCi})$ \\
\hline${ }^{13} \mathrm{~N}$-ammonia & $370-740 \mathrm{MBq}(10-20 \mathrm{mCi})$ & $\begin{array}{l}\text { Urinary bladder, } 0.0069 \mathrm{mGy} / \mathrm{MBq} \\
(0.026 \mathrm{rad} / \mathrm{mCi})\end{array}$ & $0.0022 \mathrm{mSv} / \mathrm{MBq}(0.0081 \mathrm{rem} / \mathrm{mCi})$ \\
\hline
\end{tabular}

*See package insert for full prescribing information and complete radiation dosimetry.

${ }^{\dagger}$ ICRP 53, page 373.

‡ICRP 62, page 23.

$\S$ ICRP 53, page 162

maceutical (within minutes of injection for thallium, and 30-60 min for sestamibi and tetrofosmin). Anatomic structures that may attenuate myocardial activity (i.e., breast tissue) should be positioned in identical fashion for the rest and stress studies.

2. SPECT images can be recorded with either a $180^{\circ}$ or $360^{\circ}$ collection. A $180^{\circ}$ acquisition is preferred for non-attenuation-corrected images because of better spatial resolution, higher contrast, and less attenuation. Imaging with ${ }^{201} \mathrm{Tl}$ should commence 5-10 min after injection, as soon as the patient's heart rate has returned to near baseline levels, to maximize the ability to detect transient left ventricular dysfunction. Imaging with ${ }^{99 \mathrm{~m}} \mathrm{Tc}$-sestamibi or -tetrofosmin should commence after liver activity has sufficiently cleared, usually $15-30 \mathrm{~min}$ after a stress injection and 45-60 min after a rest injection. The patient should be placed in a comfortable position on the SPECT table. The left arm should be positioned away from the field of acquisition. Data are usually recorded with the patient supine; however, in patients likely to have significant diaphragmatic (abdominal or breast) attenuation, imaging in the prone or left lateral position may produce a better result. To reduce artifacts due to attenuation, a scintillation camera with attenuation correction hardware and software may be used. Either a step-and-shoot acquisition with 32 or 64 stops separated by $3^{\circ}-6^{\circ}$ or continuous acquisition may be used. The duration of acquisition at each stop varies with the protocol and radiopharmaceutical that is used (generally 40 s/image for thallium and low-dose technetium sestamibi/tetrofosmin, and $25 \mathrm{~s} / \mathrm{image}$ for high-dose technetium sestamibi/tetrofosmin). ECG gating for the acquisition of cardiac function should be used whenever possible (particularly when the studies are recorded with ${ }^{99 \mathrm{~m}} \mathrm{Tc}$-radiopharmaceuticals) and may be accomplished with the placement of nonradiopaque electrodes and a gating device. SPECT images are acquired using a high-resolution collimator. Planar images may be acquired before the initiation of the SPECT acquisition to evaluate lung radiopharmaceutical uptake (i.e., lung-to-heart activity ratio) and to evaluate the pattern of myocardial perfusion in the event of patient movement during SPECT acquisition.

Data may be recorded with attenuation correction, using either an $\mathrm{x}$-ray or a radionuclide source. The $\mathrm{x}$-ray source is preferred, because of the reduction in noise of the attenuation correction data. The attenuation correction information reduces the influence of photon attenuation as a cause of decreased myocardial activity. Although this approach is attractive, some available hardware and software result in artifacts. As a result, both uncorrected and corrected data should be reviewed to minimize the likelihood of misinterpretation. Because misregistration of the attenuation correction map and the emission data is a potential source of artifacts, a fused image demonstrating the relation of these 2 datasets should be reviewed before evaluation of the attenuation correction emission data.

3. Positron images are generally corrected for attenuation. Because of the short physical half-life of ${ }^{82} \mathrm{Rb}$ $(\sim 75 \mathrm{~s})$ and to minimize misalignment between emission and transmission images, pharmacologic stress is used in these patients. In many PET systems, the initial acquisition is performed as a "scout" with 555-740 $\mathrm{MBq}(15-20 \mathrm{mCi})$ of ${ }^{82} \mathrm{Rb}$ to help positioning and the timing of the start of image acquisition (a longer delay in patients with slow circulation times, usually because of heart failure). The resting study can be performed first, because of the short half-life of ${ }^{82} \mathrm{Rb}$. Image acquisition usually commences 65-95 s after the intravenous administration of ${ }^{82} \mathrm{Rb}$ (longer times in patients with slow circulation times, indicated on the scout images) 
and continues for 5-10 min. Next, the transmission images are recorded to acquire 30-40 million counts, beginning about 9-10 min after injection of rubidium for the scout scan. Finally, dipyridamole or adenosine is administered intravenously, followed by an ${ }^{82} \mathrm{Rb}$ injection and PET acquisition during maximal pharmacologic stress. Myocardial perfusion imaging with ${ }^{13} \mathrm{~N}$-ammonia follows the same protocol as described for ${ }^{82} \mathrm{Rb}$. However, because of its longer physical half-life of 9.8 min, image acquisition times and temporal spacing between rest and stress radiotracer injections must be longer. Generally, 555-925 MBq (15-25 mCi) of ${ }^{13} \mathrm{~N}$-ammonia are administered, with image acquisition commencing after 4-5 min. Static images are acquired for a total of 10-15 min. The intervals between the rest and stress injections of ${ }^{13} \mathrm{~N}$-ammonia typically range from 40 to 50 min (i.e., 4-5 half lives to allow for sufficient tracer decay). In patients with prolonged circulation times or with markedly diminished left ventricular function, longer times may be needed for radiotracer clearance from blood and thus until the beginning of the image acquisition (7-8 $\mathrm{min}$ ). The relative distribution of perfusion is evaluated at rest, followed by evaluation at stress. When the patient is imaged on a PET/CT device, the scout CT scan is used for positioning. Separate attenuation-correction CT data should be recorded for the rest and stress emission images. ECG gating for the acquisition of cardiac function data should be used whenever possible.

4. The study is initially reviewed for possible artifacts due to attenuation or patient motion, image processing problems, and overall image quality before visual analysis or quantitative interpretation. Planar and SPECT images should be viewed on a computer display to permit adjustment of contrast and brightness, optimized to the myocardium. Before reconstruction, the SPECT projection data should be reviewed as a cine display to detect patient motion. Significant patient motion during image acquisition may necessitate the reprocessing or reacquisition of these studies. The data should be reconstructed using either a filtered backprojection or iterative reconstruction algorithm. Perfusion PET images should be carefully reviewed to detect cardiac displacement between rest and stress. On the ${ }^{13} \mathrm{~N}$ ammonia perfusion images, the radiotracer activity in the lateral wall of the left ventricular myocardium may be diminished in healthy volunteers, and this factor needs to be considered when patient studies are interpreted.

The orientation for the display of tomographic myocardial perfusion data is shown in Figure 1.

\section{F. Interventions}

Stress tests are described above.

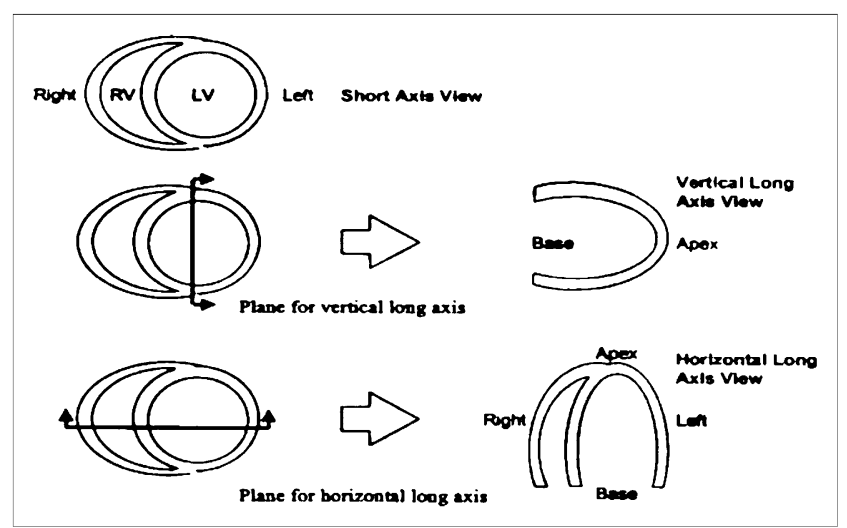

FIGURE 1. Orientation for display of tomographic myocardial perfusion data.

\section{G. Processing}

Approaches to data processing and study quality control are described in the guideline on SPECT (see SNM Procedure Guideline for General Imaging). After reconstruction, the myocardial perfusion images can be analyzed for the relative activity in each segment of myocardium and compared with a normal database. Before the data are quantified, the images should be reviewed for artifacts due to attenuation or zones of unexpected increased activity. In the absence of artifacts, the zones of myocardium for quantification are selected, the myocardial borders are defined, and the programs then calculate and display the relative distribution of activity. As with other forms of quantitation, these data are useful to supplement the interpretation of an experienced observer.

\section{H. Interpretation Criteria}

Before the images are interpreted, the data should be reviewed for artifacts due to attenuation or zones of unexpected increased activity that may alter the appearance of the myocardium. In the absence of artifacts, the images are evaluated for areas of decreased radiopharmaceutical concentration in the stress or rest images and for changes in regional count density when gated data are recorded. Zones of myocardium with tracer concentration below normal with injection at rest are usually associated with myocardial scar, but fixed defects with uptake greater than $50 \%$ of normal regions are often viable. Defects seen at stress that improve on the resting study are usually due to ischemia. Additional parameters that are particularly useful on planar thallium images are increased lung uptake or left ventricular cavity dilatation, as markers of severe left ventricular dysfunction.

\section{Viability}

${ }^{201} \mathrm{Tl}$ can be injected at rest to detect decreased perfusion to areas of viable myocardium. Images recorded 10-15 min after injection (initial images) are compared with those recorded at least 3-24 h later. An increase in the relative concentration of tracer from that seen initially to that recorded later indicates viable myocardium. An alternative 
to thallium rest/redistribution imaging compares the regional distribution of myocardial glucose use to regional myocardial perfusion. Two sets of images are recorded. The first is with ${ }^{18} \mathrm{~F}$-FDG administered under conditions of glucose loading to determine the regional myocardial use of glucose, and the second is regional myocardial perfusion determined at rest (with ${ }^{13} \mathrm{~N}$-ammonia or ${ }^{82} \mathrm{Rb}$ ). The regional distribution of perfusion and that of ${ }^{18} \mathrm{~F}-\mathrm{FDG}$ are compared. Although visual techniques may be used to compare these image sets, a more rigorous approach uses polar maps to compare the data recorded with each tracer to normal databases. A difference map comparing the relative regional distribution of ${ }^{18} \mathrm{~F}-\mathrm{FDG}$ to that of perfusion is used to identify viable ischemic tissue. Regions of perfusion that deviate by $-\geq 2$ SDs from the mean and regions of ${ }^{18}$ F-FDG uptake which deviate by $+\geq 2$ SDs identify viable ischemic tissue. It is important to consider instrumentation-related differences when viability is assessed with SPECT perfusion and PET metabolism imaging (i.e., photon attenuation-related apparent "perfusion defects" as compared with attenuation-corrected PET images of myocardial glucose uptake).

\section{J. Reporting}

The report should contain information about the indication for the study, condition of the patient at the time of injection, specific radiopharmaceutical, dose and route of administration, distribution of radiopharmaceutical in the myocardium, relative size and shape of the left ventricle, and, with a gated scan, regional thickening of the myocardium and left ventricular ejection fraction. The report should conclude with a concise impression.

Information about the condition of the patient at the time of injection should include the type of stress (e.g., treadmill or bicycle), exercise level achieved (preferably expressed in mets), heart rate, blood pressure, symptoms, brief description of the electrocardiogram at rest, and changes induced by stress. The duration of exercise should also be stated. With pharmacologic stress, the type, dose of drug, duration of infusion and symptoms, heart rate, blood pressure, and ECG changes should be noted. Institutions where the stress test and nuclear imaging are done separately may issue separate reports about each part of the procedure.

Information about the distribution of radiopharmaceutical should include the site and extent of reduced perfusion, the likely coronary vessel involved, and the relationship of regional wall thickening to abnormal perfusion.

\section{K. Quality Control}

See Guideline on General Imaging.

\section{Sources of Error}

1. Interstitial (nonintravenous) injection of the radiopharmaceutical due to a malfunctioning intravenous catheter will reduce delivery of the radiopharmaceutical to the myocardium and alter radiopharmaceutical uptake and clearance kinetics. A low-count image should raise concern regarding adequate delivery of the radiopharmaceutical. Confirmation of the infiltration by imaging of the injection site is recommended.

2. Voluntary or involuntary patient motion during image acquisition will create image blurring and artifacts that may appear as irregularities in the myocardium or as zones of decreased uptake in the myocardium. Careful attention to patient comfort and stability during the acquisition may prevent major motion artifacts. Minor motion artifacts can often be corrected by reprocessing of data, using a motion-correction algorithm. Patients should not be released before review of raw data for patient motion.

3. Failure to achieve the sex- and age-predicted $\geq 85 \%$ peak maximal heart rate will reduce the sensitivity of this procedure for detection of CAD. Patients who cannot achieve $85 \%$ of maximal predicted heart rate should be considered for pharmacologic stress before stress injection. Concomitant medications that attenuate or block the action of pharmacologic stress agents may have a similar effect.

4. Inappropriate filtering of raw back-projected tomographic data may significantly degrade image quality. When possible, iterative reconstruction should be used. Inappropriate count normalization of stress and rest images may cause noncomparability of images for diagnostic analysis.

5. Failure to recognize and account for the presence of soft-tissue attenuation (often due to the breasts, obesity, abdominal structures, etc.) can hamper accurate image analysis by creating false-positive lesions on the rest or stress images. Prone imaging, or the use of attenuation-correction hardware and software, can reduce these artifacts.

6. SNM-approved nomenclature should be used to describe anatomic areas in each of the 3 reconstructed orthogonal tomographic planes and on each of the 3 planar images to avoid diagnostic inconsistencies and render comparisons to previous studies easier. Prior studies should be reviewed for comparison to the current study to note differences (i.e., new findings) in comparison to any prior study.

7. Comparable views and tomographic slices should be displayed for comparison of the rest and stress (or redistribution) data.

8. Before examination of reconstructed tomographic cuts, the raw tomographic data should be reviewed in a rotational cinematic format for the presence of attenuation artifacts and zones of increased activity (e.g., lung, liver, bowel, or renal activity and other lesions) that may alter the appearance of the myocardium on the reconstructed data. If possible, steps should be taken to compensate for these problems, or the acquisition may have to be repeated. 
9. For quantitative analysis of regional myocardial and lung activity, it is necessary to ensure that regions of interest do not include activity from adjacent structures. Calculation of the lung-to-heart activity ratio should include a similarly sized region of interest in the lung and myocardium, not including the anterior and anterolateral wall, where lung and myocardium activity overlap. Attempts should be made to include only cardiac activity in regions of interest used for quantitative analysis of radiopharmaceutical uptake and clearance.

10. For quantitative analysis, the SPECT data must be processed in an identical fashion to the normal data file, including filtering, reorientation, and quantitative analysis.

\section{ISSUES REQUIRING FURTHER CLARIFICATION}

None.

\section{CONCISE BIBLIOGRAPHY}

A. ACC/AHA/ASNC guidelines for the clinical use of cardiac radionuclide imaging: executive summary-a report of the American College of Cardiology/American Heart Association Task Force on Practice Guidelines (ACC/AHA/ASNC Committee to Revise the 1995 Guidelines for the Clinical Use of Cardiac Radionuclide Imaging). J Am Coll Cardiol. 2003; 42:1318-1333.

B. ACCF/ASNC appropriateness criteria for singlephoton emission computed tomography myocardial perfusion imaging (SPECT MPI): a report of the American College of Cardiology Foundation Quality Strategic Directions Committee Appropriateness Criteria Working Group and the American Society of Nuclear Cardiology endorsed by the American Heart Association. J Am Coll Cardiol. 2005;46: 1587-1605.

C. Imaging guidelines for nuclear cardiology procedures. J Nucl Cardiol. 2006;13:e21-e171.

\section{DISCLAIMER}

The SNM has written and approved this Procedure Guideline as an educational tool designed to promote the costeffective use of high-quality nuclear medicine procedures in medical practice or in the conduct of research and to assist practitioners in providing appropriate care for patients. The Procedure Guideline should not be deemed inclusive of all proper procedures or exclusive of other procedures reasonably directed to obtaining the same results. The guidelines are neither inflexible rules nor requirements of practice and are not intended nor should they be used to establish a legal standard of care. For these reasons, the SNM cautions against the use of this Procedure Guideline in litigation in which the clinical decisions of a practitioner are called into question.

The ultimate judgment about the propriety of any specific procedure or course of action must be made by the physician when considering the circumstances presented. Therefore, an approach that differs from the Procedure Guideline is not necessarily below the standard of care. A conscientious practitioner may responsibly adopt a course of action different from that set forth in the Procedure Guideline when, in his or her reasonable judgment, that course of action is indicated by the condition of the patient, limitations on available resources, or advances in knowledge or technology subsequent to publication of the Procedure Guideline.

All that should be expected is that the practitioner will follow a reasonable course of action based on current knowledge, available resources, and the needs of the patient to deliver effective and safe medical care. The sole purpose of this Procedure Guideline is to assist practitioners in achieving this objective.

Advances in medicine occur at a rapid rate. The date of a Procedure Guideline should always be considered in determining its current applicability.

\section{APPROVAL}

This Procedure Guideline was approved by the Board of Directors of the SNM on June 14, 2008. 